\title{
Performance of Pediatric Readmission Measures
}

\author{
Katherine A Auger, MD, MSc 1,2,3* Michael C Ponti-Zins ${ }^{4}$, Angela M Statile, MD, MEd $d^{1,2,3}$, Kris Wesselkamper, MD³, \\ Beth Haberman, MD ${ }^{3,6}$, Samuel P Hanke, MD, MS, MBA $2,3,4,7$
}

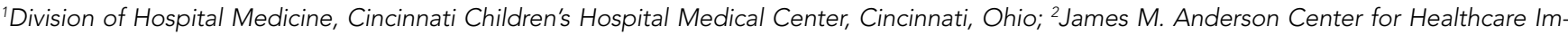
provement, Cincinnati Children's Hospital Medical Center, Cincinnati, Ohio; ${ }^{3}$ University of Cincinnati School of Medicine, Cincinnati, Ohio; ${ }^{4} \mathrm{Center}$ for Patient Family Experience, Cincinnati Children's Hospital Medical Center, Cincinnati, Ohio; ${ }^{5}$ Division of Neurology, Cincinnati Children's Hospital Medical Center, Cincinnati, Ohio; ${ }^{6}$ Perinatal Institute, Cincinnati Children's Hospital Medical Center, Cincinnati, Ohio; ${ }^{7}$ Heart Institute, Cincinnati Children's Hospital Medical Center, Cincinnati, Ohio.
\end{abstract}

BACKGROUND: Readmission rates are frequently used as a hospital quality metric; yet multiple measures exist to evaluate pediatric readmission rates. We sought to assess how four different measures of pediatric readmission compare with assessment of both preventable and unplanned readmission.

METHODS: Clinicians on hospital medicine, cardiology, neonatology, and neurology teams reviewed medical records for 30-day readmissions using an abstraction tool with high interrater reliability for preventability assessment. Readmissions between July 2014 and June 2016 were classified separately as preventable or not preventable and planned or unplanned. We compared the classifications to four existing readmission metrics: all-cause readmission, unplanned readmission/time flag classification, the pediatric all-condition readmission, and potentially preventable readmission. We calculated sensitivity and specificity for all readmission metrics.
RESULTS: Among 30-day readmissions considered, 1,643 were eligible for medical record review; 1,125 reviews were completed by the clinical teams (68\%). On medical record review, the majority of readmissions were determined not preventable (85\%). Only $15 \%$ were classified as unplanned and preventable. None of the four readmission measures had appropriate sensitivity or specificity for identifying preventable readmission. The unplanned readmission/time flag classification had the highest sensitivity (95\%) and specificity (90\%) in identifying unplanned readmissions.

CONCLUSION: None of the existing pediatric readmission measures can reliably determine preventability. The unplanned readmission/time flag measure performed best in identifying unplanned readmissions. Journal of Hospital Medicine 2020;15:723-726. (c) 2020 Society of Hospital Medicine eadmission rates are frequently used as a hospital quality metric, with use including payment incentive at the hospital level, ${ }^{1}$ specific condition quality measurement, ${ }^{2}$ balancing measures for quality improvement projects, ${ }^{3-5}$ transition success, ${ }^{6,7}$ and use in public hospital rankings. ${ }^{8}$ Currently, four methods are commonly used to evaluate pediatric readmissions, each with strengths and limitations, including the following (Appendix Table 1):

1. All-cause readmissions: A measure of any readmission within a given time period regardless of the reason for readmission. ${ }^{9}$

2. Unplanned readmission/time flag: A measure intended to identify unplanned readmissions. This measure relies on time designations within the electronic health record. The time between hospital registration and admission is calculated, and if the readmission is registered more than 24 hours prior to

*Corresponding Author: Katherine A Auger, MD; Email: katherine.auger@ cchmc.org; Telephone: 513-803-8092; Twitter: @KathyAugerpeds.

Published online first November 18, 2020.

Find additional supporting information in the online version of this article.

Received: March 2, 2020; Revised: August 5, 2020; Accepted: August 10, 2020

๑ 2020 Society of Hospital Medicine DOI 10.12788/jhm.3521 admission, the readmission is considered planned. ${ }^{10}$ Hereafter, this measure will be referred to as the time flag measure.

3. Pediatric all-condition readmission (PACR): A measure intended to identify unplanned readmission through the exclusion of certain procedures and diagnoses. ${ }^{11}$

4. Potentially preventable readmission (PPR): A method to identify preventable readmissions based on a proprietary algorithm developed by $3 \mathrm{M}$ Health Information Systems. ${ }^{12,13}$

While all four of these measures are used to assess quality, there is little known about these measures' ability to exclude planned readmissions and identify only preventable pediatric readmission, which conceptually is most relevant to the quality of care. However, many of these measures were not intended to capture preventability, but instead capture the related issue of whether the readmission was planned. Therefore, we sought to evaluate the four readmission measures as they relate to both preventability and unplanned status as determined through medical record review with multidisciplinary care provider input.

\section{METHODS}

As part of a hospital-wide readmission reduction quality improvement collaborative at a free-standing tertiary care children's 
TABLE. Sensitivity and Specificity of Preventable and Unplanned Readmission Metrics

\begin{tabular}{|c|c|c|c|c|c|}
\hline & & \multicolumn{4}{|c|}{ Sensitivity and Specificity of Preventable and Unplanned Readmission Metrics } \\
\hline \multirow{2}{*}{$\begin{array}{l}\text { A. All-Cause } \\
\mathrm{N}=1125\end{array}$} & Yes & 174 & 951 & 885 & 227 \\
\hline & No & 0 & 0 & 0 & 0 \\
\hline \multicolumn{2}{|c|}{ Sensitivity and specificity } & Sensitivity $=100 \%$ & Specificity $=0 \%$ & Sensitivity $=100 \%$ & Specificity $=0 \%$ \\
\hline \multirow{2}{*}{\multicolumn{2}{|c|}{ Estimated PPV and NPV based on varying prevalence ${ }^{b}$}} & PPV@20\% = 20\% & NPV@20\%= N/A & PPV@65\%=65\% & NPV@65\%=N/A \\
\hline & & PPV@30\%=30\% & $N P V @ 30 \%=N / A$ & PPV@70\%=70\% & NPV@70\% = N/A \\
\hline \multirow{2}{*}{$\begin{array}{l}\text { B. Time flag measure } \\
N=1125\end{array}$} & Unplanned & 158 & 716 & 843 & 23 \\
\hline & Planned & 16 & 235 & 42 & 204 \\
\hline \multicolumn{2}{|c|}{ Estimated PPV and NPV based on varying prevalence ${ }^{b}$} & PPV@30\% = 34.1\% & $\mathrm{NPV} @ 30 \%=86.2 \%$ & PPV@70\%=95.6\% & NPV@70\% = 89.0\% \\
\hline \multirow{2}{*}{$\begin{array}{l}\text { C. PACR } \\
N=804\end{array}$} & Qualifying readmission & 114 & 491 & 543 & 56 \\
\hline & Not qualifying & 17 & 182 & 104 & 93 \\
\hline \multicolumn{2}{|c|}{ Sensitivity and specificity } & Sensitivity $=87.0 \%$ & Specificity $=27.0 \%$ & Sensitivity $=83.9 \%$ & Specificity $=62.4 \%$ \\
\hline \multirow{3}{*}{\multicolumn{2}{|c|}{ Estimated PPV and NPV based on varying prevalence ${ }^{b}$}} & PPV@10\%=11.7\% & NPV@10\%=94.9\% & PPV@60\%=77.0\% & NPV@60\%=72.1\% \\
\hline & & PPV@20\%=23.0\% & NPV@20\%=89.3\% & PPV@65\%=80.6\% & NPV@65\%=67.6\% \\
\hline & & PPV@30\%=33.8\% & NPV@30\%=82.9\% & PPV@70\%=83.9\% & NPV@70\%=62.5\% \\
\hline $\begin{array}{l}\text { D. PPR } \\
N=487\end{array}$ & Preventable & 53 & 204 & 239 & 17 \\
\hline
\end{tabular}

a13 readmissions are missing from planned/unplanned determination by chart review.

bThe provided estimates of PPV and NPV are based on different plausible range of prevalence of readmission type with sensitivity and specificity kept constant. For preventable readmissions, the prevalence ranges presented are between $10 \%$ and $30 \%$; for unplanned readmissions, the prevalence is between $60 \%$ and $70 \%$ of all readmissions. PPV and NPV cannot be calculated (not applicable, N/A) when there are zero readmissions in the "No" category of the metric.

Abbreviations: NPV, negative predictive value; PACR, pediatric all-condition readmission; PPR, potentially preventable readmission, based on $3 \mathrm{M}$ Health Information Systems software; PPV, positive predictive value.

hospital, clinicians from hospital medicine, cardiology, neonatology, and neurology teams reviewed 30-day readmissions using a standardized abstraction tool. All readmission events (observation or inpatient encounter) after any discharge (observation or inpatient encounter) from eligible units were reviewed; therefore, each hospitalization was a potential index hospitalization. We classified the preventability of each readmission with use of a previously described Likert scale with high interrater reliability. ${ }^{14}$ For these analyses, readmissions were considered preventable if the reviewing team rated them as either "more likely preventable" or "preventable in most circumstances." Each readmission was also evaluated as planned or unplanned. Methods for readmission review and classification are in the Appendix.

We included all readmissions between July 2014 and June 2016. We compared the medical record review classifications with the assessments from each of the four measures of pediatric readmission. We calculated sensitivity and specificity for both outcomes (planned/unplanned and preventable/not preventable) for all four measures. For standardization of discussion, we categorized description of measure performance as "very poor" as less than 50\%, "poor" between 50\%-75\%, "fair" as 75\%-85\%, "good" as 85\%-90\%, "very good" as $90 \%-95 \%$ and excellent as greater than $95 \%$. We also calculated positive and negative predictive value (PPV and NPV) over plausible ranges of prevalence using the sensitivity and specificity of each comparison (Appendix).

Of note, certain exclusions are outlined by the PACR and PPR algorithms. The PACR evaluates only readmission events that occur in children younger than 18 years. The PPR algorithm does not assign preventability if either the index or read- 
mission event is classified as an observation stay or if it is part of a larger chain of readmissions.

\section{RESULTS}

Among 30-day readmissions considered, 1,643 were eligible for medical record review; 1,125 reviews were completed by the clinical teams (68.5\%). The median time to readmission was 7 days (interquartile range [IOR], 4-18). Most children were non-Hispanic White (71\%) or Black (20\%). The median age at hospitalization was 2.3 years (IOR 0.4-12.1). Most children had Medicaid (56\%) or private (41\%) insurance. Most of the reviews were performed in cardiology (43\%) and hospital medicine (37\%) with patients in neurology (13\%) and neonatology (7\%) constituting the remaining reviews. Uncontrolled advancement of chronic disease was the most common readmission category on medical record review (25.1\%), followed by unrelated readmission (20.7\%), scheduled readmission (20.4\%), and progression of acute disease (16.6\%) (Appendix Table 2).

\section{Assessment of Preventable and Unplanned Readmissions}

On multidisciplinary medical record review, most readmissions were classified as not preventable (84.5\%). Specifically, $64 \%$ were not preventable and unplanned; $20 \%$ were deemed not preventable and planned. Only $15 \%$ were classified as unplanned and preventable and $1 \%$ as planned and preventable (Appendix Figure: Population A/B).

\section{Matching Chart Review to the Four Algorithms}

All 1,125 readmissions were assessed by the all-cause and time flag readmission measures (Appendix Figure: Population A/B). After applying algorithm exclusions (details in Appendix), only 804 of the 1,125 (71.5\%) reviewed readmissions matched for PACR readmission comparison (Appendix Figure: Population C); 487 of the 1,125 (43.3\%) of the reviewed readmissions matched for PPR comparison (Appendix Figure: Population D).

\section{All-Cause}

Because all-cause determines only if a readmission occurs, the measure is by definition $100 \%$ sensitive and $0 \%$ specific in both assessment of preventability and unplanned readmission ( $\mathrm{Ta}$ ble: Section A).

\section{Time Flag}

The time flag measure identified $80 \%(866 / 1,112)$ of the readmissions as unplanned. This measure had very good sensitivity but very poor specificity in identifying preventable readmissions, which corresponded to very poor PPV and good to excellent NPV. In terms of identifying unplanned readmissions, the time flag measure had excellent sensitivity and very good specificity, which corresponded to very good to excellent PPV and good to very good NPV (Table: Section B).

\section{PACR}

The PACR algorithm identified 75\% (599/796) of readmissions as unplanned. The PACR has good sensitivity but very poor specificity in identifying preventable readmissions, which corresponded to very poor PPV and fair to very good NPV. In terms of identifying unplanned readmissions, the PACR had fair sensitivity but poor specificity, which corresponded to fair PPV and poor NPV (Table: Section C).

\section{PPR}

The PPR algorithm identified 53\% (257/487) of admissions as potentially preventable. The PPR algorithm had poor sensitivity and specificity in identifying preventable readmissions, which corresponded to very poor PPV and fair to very good NPV. In terms of identifying unplanned readmissions, the PPR algorithm had poor sensitivity and fair specificity in identifying unplanned readmissions, which corresponded to fair to good PPV and very poor to poor NPV (Table: Section D).

\section{Evaluation of Excluded Readmission Events}

Because both the PACR and PPR had large numbers of algorithm exclusions, we describe the preventability and unplanned assessment of the excluded readmission events. Both algorithms excluded preventable events. Of the 321 readmissions excluded by the PACR algorithm, $13.4 \%$ were classified as preventable by chart review. Likewise, $14.9 \%$ of 638 readmissions excluded by PPR were classified as preventable by chart review.

\section{DISCUSSION}

The ability to accurately capture preventable pediatric readmission is a goal for hospital quality experts and health policymakers alike. Of the four commonly used readmission measures to assess readmission, only PPR is designed to focus on preventability. Unfortunately, none of these four measures is adequately sensitive or specific to identify preventable readmissions; all measures had very poor PPV for preventability. Of the four measures, the time flag measure had the best sensitivity, specificity, PPV, and NPV for identifying unplanned readmissions.

The overall percentage of unplanned readmissions identified by both the time flag and by PACR measures match the overall percentage of unplanned readmissions identified in chart review: The time flag measure identified $80 \%$ of admissions as unplanned versus 79\% identified by chart review (Appendix Figure: Population A/B); PACR classified 75\% as unplanned versus $81 \%$ identified by chart review for PACR-eligible readmissions (Appendix Figure: Population C). In contrast, the PPR algorithm classified many more readmissions as potentially preventable (53\%) than were identified by chart review at only 16\% (Appendix Figure: Population D). The PACR and PPR algorithms also exclude a significant number of readmissions that are unplanned and a smaller, but not trivial, number of readmissions that are preventable; these exclusions limit their accuracy.

The ability to apply these four measures in real time during a hospitalization varies by metric. Two of the measures, the all-cause and time flag, can be applied during a readmission event, which is appealing for quality improvement initiatives. 
These measures allow for notification of providers that a current hospitalization is a readmission event, which allows providers the opportunity to learn from these events as they occur (Appendix Table 1). While "unplanned" is not the same as "potentially preventable," almost all potentially preventable readmissions are unplanned; therefore, accurately identifying unplanned readmissions is more beneficial than all-cause. Additionally, a low all-cause readmission rate can be indicative of poor access to scheduled procedures. Nevertheless, all-cause readmission is sometimes used to measure quality. ${ }^{1,8}$ While the time flag measure may be more useful for quality improvement initiatives and hospital providers, it relies on hospital registration time, which is not widely available in administrative data sources and, therefore, has limited usefulness to policymakers.

Both PACR and PPR require administrative claims analysis, which is appealing from a policy standpoint. However, the reliance on claims data means the inclusion/exclusion of events can occur only retrospectively, which limits the usefulness of these measures in learning and intervening in real time. When the two measures are compared, PACR offers better sensitivity and PPR offers better specificity with regard to identifying unplanned readmission. The PPR software overcalls preventable readmissions, identifying more readmissions as preventable than there actually are. Nevertheless, Medicaid in several states uses PPR for payment incentive. ${ }^{1,15-17}$ Given the poor performance of PPR in assessing both preventable and unplanned pediatric readmission, the use of this measure as a quality metric should be limited.

This study should be considered in the context of several limitations. Because the assessment of preventability was determined as part of a learning quality improvement collaborative and not as a planned research endeavor, not all readmis-

\section{References}

1. State Medicaid Payment Policies for Inpatient Hospital Services. Medicaid and CHIP Payment and Access Commission; December 2018. Accessed June 1, 2019. https://www.macpac.gov/publication/macpac-inpatient-hospital-payment-landscapes/

2. Mangione-Smith R, Zhou C, Williams DJ, et al. Pediatric Respiratory IIIness Measurement System (PRIMES) scores and outcomes. Pediatrics. 2019;144(2):e20190242. https://doi.org/10.1542/peds.2019-0242

3. Biondi EA, McCulloh $R$, Staggs VS, et al. Reducing Variability in the Infant Sepsis Evaluation (REVISE): a national quality initiative. Pediatrics. 2019;144(3):e20182201. https://doi.org/10.1542/peds.2018-2201

4. Statile AM, Schondelmeyer AC, Thomson JE, et al. Improving discharge efficiency in medically complex pediatric patients. Pediatrics. 2016;138(2):e20153832. https://doi.org/10.1542/peds.2015-3832

5. White CM, Statile AM, White DL, et al. Using quality improvement to optimise paediatric discharge efficiency. BMJ Qual Saf. 2014;23(5):428-436. https://doi.org/10.1136/bmjqs-2013-002556

6. Auger KA, Simmons JM, Tubbs-Cooley HL, et al; H2O Trial Study Group. Postdischarge nurse home visits and reuse: the Hospital to Home Outcomes (H2O) trial. Pediatrics. 2018;142(1):e20173919. https://doi.org/10.1542/peds.2017-3919

7. Auger KA, Shah SS, Tubbs-Cooley HL, et al. Effects of a 1-time nurse-led telephone call after pediatric discharge: the $\mathrm{H} 2 \mathrm{O}$ II randomized clinical trial. JAMA Pediatr. 2018;172(9):e181482. https://doi.org/10.1001/jamapediatrics.2018.1482

8. Olmsted MG, Powell R, Murphy J, Bell Denise, Stanley M, Sanchz R. Methodology: U.S. News \& World Report Best Children's Hospitals 2019-20. U.S News \& World Report; June 17, 2019. Accessed June 16, 2020. https://www usnews.com/static/documents/health/best-hospitals/BCH_Methodology_2019-20.pdf

9. Bardach NS, Vittinghoff E, Asteria-Peñaloza R, et al. Measuring hospital quality using pediatric readmission and revisit rates. Pediatrics. 2013;132(3):429436. https://doi.org/10.1542/peds.2012-3527 sion reviews were completed nor were other existent tools ${ }^{18}$ that allow for preventability assessment via more structured medical record review used. Second, we reviewed cases only from certain clinical services, which would limit generalizability of these findings to all pediatric admissions. However, given the low sensitivity and specificity of some of the metrics, we would not anticipate that the addition of other types of admissions would improve the sensitivity and specificity enough to ensure reliability. Third, while we relied on an established method to determine preventability, prior work has demonstrated that additional information gathered from families may change preventability. ${ }^{19}$ Finally, due to the exclusions required by the PPR and PACR algorithms, not all readmission events were reviewed. However, these exclusions reflect the actual specifications of use for both measures.

\section{CONCLUSION}

The PPR software has poor fidelity in identifying preventable and unplanned pediatric readmission; this finding has broad policy implications given how widely it is used by state Medicaid offices to assess financial penalties. Among the four pediatric readmission measures used, the time flag metric best identifies unplanned readmissions.

Disclosures: The authors have no conflicts of interest or financial relationships relevant to this article to disclose.

Funding: Dr Auger's research is supported by a grant from the Agency for Healthcare Research and Quality (1K08HS204735-01A1). The project described was supported by the National Center for Advancing Translational Sciences of the National Institutes of Health, under Award Number 5UL1TR001425-04 The content is solely the responsibility of the authors and does not necessarily represent the official views of the $\mathrm{NIH}$

10. Auger KA, Mueller EL, Weinberg $\mathrm{SH}$, et al. A validated method for identifying unplanned pediatric readmission. J Pediatr. 2016;170:105-12.e102. https://doi.org/10.1016/j.jpeds.2015.11.051

11. Readmissions-Content. Boston Children's Hospital. Accessed April 8 2019. http://www.childrenshospital.org/research-and-innovation/research/ centers/center-of-excellence-for-pediatric-quality-measurement-cepqm/ cepqm-measures/pediatric-readmissions/content

12. Gay JC, Agrawal R, Auger KA, et al. Rates and impact of potentially preventable readmissions at children's hospitals. J Pediatr. 2015;166(3):613-9.e5. https://doi.org/10.1016/j.jpeds.2014.10.052

13. Auger KA, Teufel RJ, Harris JM, et al. Children's hospital characteristics and readmission metrics. Pediatrics. 2017;139(2):e20161720. https://doi. org/10.1542/peds.2016-1720

14. Hain PD, Gay JC, Berutti TW, Whitney GM, Wang W, Saville BR. Preventability of early readmissions at a children's hospital. Pediatrics. 2013;131(1):e171-e181. https://doi.org/10.1542/peds.2012-0820

15. Potentially Preventable Events. Texas Health and Human Services. Accessed May 19, 2019. https://hhs.texas.gov/about-hhs/process-improvement/medicaid-chip-quality-efficiency-improvement/potentially-preventable-events

16. Potentially Preventable Readmissions. New York State Department of Health. Accessed May 28, 2019. https://regs.health.ny.gov/sites/default/files/pdf/recently_adopted_regulations/2011-02-23_potentially_preventable_readmissions.pdf

17. Potentially Preventable Readmissions Policy. Illinois Department of Healthcare and Family Services. Accessed May 28, 2019. https://www.illinois.gov/ $\mathrm{hfs} /$ SiteCollectionDocuments/PPR_Overview.pdf

18. Jonas JA, Devon EP, Ronan JC, et al. Determining preventability of pediatric readmissions using fault tree analysis. J Hosp Med. 2016;11(5):329-335. https://doi.org/10.1002/jhm.2555

19. Toomey SL, Peltz A, Loren S, et al. Potentially preventable 30-day hospital readmissions at a children's hospital. Pediatrics. 2016;138(2):e20154182. https://doi.org/10.1542/peds.2015-4182 